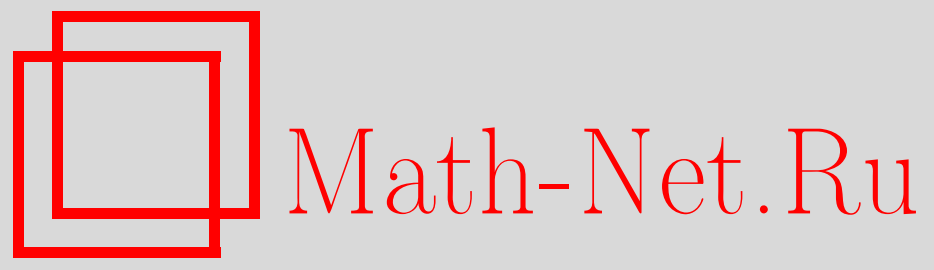

S. Nadarajah, S. Kotz, A Class of $t$ Posterior Distributions with Application, Теория вероятн. и ее примен., 2008, том 53, выпуск 1, 200-207

DOI: https://doi.org/10.4213/tvp2495

Использование Общероссийского математического портала Math-Net.Ru подразумевает, что вы прочитали и согласны с пользовательским соглашением

http: //www.mathnet.ru/rus/agreement

Параметры загрузки:

IP : 18.207 .199 .55

26 апреля 2023 г., 10:23:59

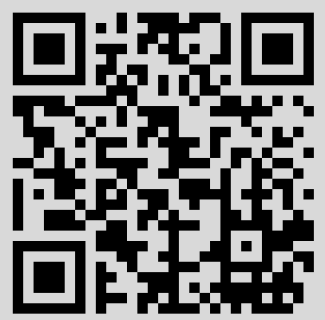


28. Ядренко М. Й. Спектральная теория случайных полей. Киев: Виша школа, 1980, $208 \mathrm{c}$.

29. Yaglom A.M. Correlation Theory of Stationary and Related Random Functions, v. 1, 2. New York: Springer, 1987, 526 p.; 258 p.

30. Zastavnyi V.P. On positive definiteness of some functions. - J. Multivariate Anal., 2000 , v. 73 , № 1 , p. $55-81$.

Поступила в редакцию

28.XII. 2007

(C) 2008 г

NADARAJAH S.*, KOTZ S.**

\section{A CLASS OF $t$ POSTERIOR DISTRIBUTIONS WITH APPLICATION}

Устанавливается класс апостериорных распределений параметра положения для некоторых генеральных совокупностей. В качестве априорного распределения для параметра масштаба берется распределение Стьюдента. В вычислениях задействованы некоторые специальные функции. Дается применение к описанию распределений коэффициентов дискретного косинус-преобразования.

Ключевые слова и фразы: дискретное косинус-преобразование, параметр положения, апостериорное распределение, распределение Стьюдента.

1. Introduction. Suppose we have a population and that we wish study the distribution of its mean. The most commonly encountered situation is the study of the mean of a normal population. The fashionable approach these days is to use Bayesian statistics, i.e., try to derive the posterior distribution of the population mean based on some available information.

Let $g(\cdot ; \lambda, \mu)$ denote the probability density function (pdf) of the observed values of the desired population. Here, $\mu$ and $\lambda$ denote some location and scale parameters, respectively, representing the population mean and population variance, respectively. For example, if $g$ is a normal pdf, then $\mu$ is the population mean and $\lambda$ is the population standard deviation.

Usually, the observer will have some prior knowledge about the scale parameter $\lambda$. For the past 40 to 50 years, the Student's $t$ distribution has been the most popular prior distribution because elicitation of prior information in various physical, engineering, and financial phenomena is closely associated with that distribution, see [2]. So, if we assume that

$$
p(\lambda) \propto\left(1+\frac{\lambda^{2}}{\nu}\right)^{-(1+\nu) / 2}
$$

then the joint posterior will be

$$
p(\mu, \lambda \mid x) \propto g(x ; \lambda, \mu)\left(1+\frac{\lambda^{2}}{\nu}\right)^{-(1+\nu) / 2}
$$

Hence, the marginal posterior of $\mu$ will be

$$
p(\mu \mid x) \propto \int_{0}^{\infty} g(x ; \lambda, \mu)\left(1+\frac{\lambda^{2}}{\nu}\right)^{-(1+\nu) / 2} d \lambda
$$

* School of Mathematics, University of Manchester, Manchester M60 1QD, UK; e-mail: saralees.nadarajah@manchester.ac.uk

** Department of Engineering Management and Systems Engineering, George Washington University, Washington, D.C. 20052, USA 
The density in (1) is the same as that of $\mu+X Y$ when $X \sim g(\cdot ; 1,0)$ and $Y \sim$ Student's $t$ are distributed independently of each other. Hence, calculating the marginal posterior of $\mu$ amounts to deriving the exact distribution of $X Y$.

In this note, we derive the exact distribution of $X Y$ or equivalently the marginal posterior of $\mu$ with $g(\cdot ; \lambda, \mu)$ taken to be one of the following: normal pdf (Section 2), Student's $t$ pdf (Section 3), Laplace pdf (Section 4), logistic pdf (Section 5), and Bessel function pdf (Section 6). For each of these cases, the exact distribution of $X Y$ is expressed in terms of its characteristic function, i.e.,

$\phi(t)=\mathbf{E}[\exp (i t X Y)]=\frac{1}{\sqrt{\nu} \mathrm{B}(\nu / 2,1 / 2)} \int_{-\infty}^{\infty} \int_{-\infty}^{\infty} \exp (i t x y)\left(1+\frac{x^{2}}{\nu}\right)^{-(1+\nu) / 2} g(y) d x d y$.

An application of the results in Sections 2-6 to DCT coefficients is described in Section 7.

The calculations of this note involve several special functions, including the ${ }_{1} F_{1}$ hypergeometric function (also known as the confluent hypergeometric function) defined by

$$
{ }_{1} F_{1}(a ; b ; x)=\sum_{k=0}^{\infty} \frac{(a)_{k}}{(b)_{k}} \frac{x^{k}}{k !},
$$

the Kummer function defined by

$$
\Psi(a, b ; x)=\frac{\Gamma(1-b){ }_{1} F_{1}(a ; b ; x)}{\Gamma(1+a-b)}+\frac{\Gamma(b-1) x^{1-b}{ }_{1} F_{1}(1+a-b ; 2-b ; x)}{\Gamma(a)},
$$

the ${ }_{1} F_{2}$ hypergeometric function defined by

$$
{ }_{1} F_{2}(a ; b, c ; x)=\sum_{k=0}^{\infty} \frac{(a)_{k}}{(b)_{k}(c)_{k}} \frac{x^{k}}{k !},
$$

the ${ }_{2} F_{1}$ hypergeometric function (also known as the Gauss hypergeometric function) defined by

$$
{ }_{2} F_{1}(a, b ; c ; x)=\sum_{k=0}^{\infty} \frac{(a)_{k}(b)_{k}}{(c)_{k}} \frac{x^{k}}{k !},
$$

and the modified Bessel function of the third kind defined by

$$
K_{\nu}(x)=\frac{\sqrt{\pi} x^{\nu}}{2^{\nu} \Gamma(\nu+1 / 2)} \int_{1}^{\infty}\left(t^{2}-1\right)^{\nu-1 / 2} \exp (-x t) d t
$$

where $(e)_{k}=e(e+1) \cdots(e+k-1)$ denotes the ascending factorial. The properties of the above special functions can be found in [4] and [1].

2. Normal $g$. If $g$ is the normal pdf given by

$$
g(y)=\frac{1}{\sqrt{2 \pi} \sigma} \exp \left\{-\frac{(y-\mu)^{2}}{2 \sigma^{2}}\right\},
$$

then (2) can be reduced as

$$
\begin{aligned}
\phi(t)= & \frac{1}{\sqrt{\nu} B(\nu / 2,1 / 2)} \frac{1}{\sqrt{2 \pi} \sigma} \int_{-\infty}^{\infty} \int_{-\infty}^{\infty}\left(1+\frac{x^{2}}{\nu}\right)^{-(1+\nu) / 2} \exp \left\{i t x y-\frac{(y-\mu)^{2}}{2 \sigma^{2}}\right\} d y d x \\
= & \frac{1}{\sqrt{\nu} \mathrm{B}(\nu / 2,1 / 2)} \frac{1}{\sqrt{2 \pi} \sigma} \\
& \times \int_{-\infty}^{\infty} \int_{-\infty}^{\infty}\left(1+\frac{x^{2}}{\nu}\right)^{-(1+\nu) / 2} \exp \left\{\frac{2 \sigma^{2} i t x y-y^{2}+2 \mu y-\mu^{2}}{2 \sigma^{2}}\right\} d y d x \\
= & \frac{1}{\sqrt{\nu} \mathrm{B}(\nu / 2,1 / 2)} \frac{1}{\sqrt{2 \pi} \sigma} \int_{-\infty}^{\infty} \int_{-\infty}^{\infty}\left(1+\frac{x^{2}}{\nu}\right)^{-(1+\nu) / 2} \\
& \times \exp \left\{-\frac{\left(y-\mu-\sigma^{2} i t x\right)^{2}}{2 \sigma^{2}}+\mu i t x-\frac{\sigma^{2} t^{2} x^{2}}{2}\right\} d y d x
\end{aligned}
$$




$$
\begin{aligned}
& =\frac{1}{\sqrt{\nu} \mathrm{B}(\nu / 2,1 / 2)} \int_{-\infty}^{\infty}\left(1+\frac{x^{2}}{\nu}\right)^{-(1+\nu) / 2} \exp \left(\mu i t x-\frac{\sigma^{2} t^{2} x^{2}}{2}\right) d x \\
& =\frac{2}{\sqrt{\nu} \mathrm{B}(\nu / 2,1 / 2)} \int_{0}^{\infty}\left(1+\frac{x^{2}}{\nu}\right)^{-(1+\nu) / 2} \cos (\mu t x) \exp \left(-\frac{\sigma^{2} t^{2} x^{2}}{2}\right) d x
\end{aligned}
$$

The integral in (3) cannot be calculated directly in its general form. However, using the series expansion for cosine,

$$
\cos (x)=\sum_{k=0}^{\infty} \frac{(-1)^{k} x^{2 k}}{(2 k) !}
$$

one can rewrite (3) as

$$
\begin{aligned}
\phi(t) & =\frac{2}{\sqrt{\nu} \mathrm{B}(\nu / 2,1 / 2)} \int_{0}^{\infty}\left(1+\frac{x^{2}}{\nu}\right)^{-(1+\nu) / 2}\left\{\sum_{k=0}^{\infty} \frac{(-1)^{k}(\mu t x)^{2 k}}{(2 k) !}\right\} \exp \left(-\frac{\sigma^{2} t^{2} x^{2}}{2}\right) d x \\
& =\frac{2 \nu^{\nu / 2}}{\mathrm{~B}(\nu / 2,1 / 2)} \sum_{k=0}^{\infty} \frac{(-1)^{k}(\mu t)^{2 k}}{(2 k) !} \int_{0}^{\infty} x^{2 k}\left(\nu+x^{2}\right)^{-(1+\nu) / 2} \exp \left(-\frac{\sigma^{2} t^{2} x^{2}}{2}\right) d x \\
& =\frac{\nu^{\nu / 2}}{\mathrm{~B}(\nu / 2,1 / 2)} \sum_{k=0}^{\infty} \frac{(-1)^{k}(\mu t)^{2 k}}{(2 k) !} \int_{0}^{\infty} w^{k-1 / 2}(\nu+w)^{-(1+\nu) / 2} \exp \left(-\frac{\sigma^{2} t^{2} w}{2}\right) d w,(5)
\end{aligned}
$$

where the last step follows by the substitution $w=x^{2}$. The integral in (5) can be calculated by direct application of equation (2.3.6.9) in [4, v. 1]) to yield

$$
\phi(t)=\frac{1}{\mathrm{~B}(\nu / 2,1 / 2)} \sum_{k=0}^{\infty} \frac{(-1)^{k} \nu^{k}(\mu t)^{2 k}}{(2 k) !} \Gamma\left(k+\frac{1}{2}\right) \Psi\left(k+\frac{1}{2}, k-\frac{\nu}{2}+1 ; \frac{\sigma^{2} t^{2} \nu}{2}\right),
$$

an infinite sum involving the Kummer function. If $\mu=0$, then (6) reduces to the simple form

$$
\phi(t)=\frac{\Gamma((\nu+1) / 2)}{\Gamma(\nu / 2)} \Psi\left(\frac{1}{2}, \frac{2-\nu}{2} ; \frac{\sigma^{2} t^{2} \nu}{2}\right) .
$$

If $\mu=0$ and $\nu=1$, then (7) can be reduced further to

$$
\phi(t)=\exp \left(\frac{\sigma^{2} t^{2}}{2}\right) \operatorname{erfc}\left(\frac{\sigma|t|}{\sqrt{2}}\right) .
$$

3. Student's $t g$. Suppose $g$ is the Student's $t$ pdf with degrees of freedom $b$ given by

$$
g(y)=\frac{1}{\sqrt{b} \mathrm{~B}(b / 2,1 / 2)}\left(1+\frac{y^{2}}{b}\right)^{-(1+b) / 2} .
$$

Note that (2) can be expressed as

$$
\begin{aligned}
\phi(t) & =\frac{1}{\sqrt{\nu} \mathrm{B}(\nu / 2,1 / 2)} \int_{-\infty}^{\infty} \int_{-\infty}^{\infty} \exp (\text { it xy })\left(1+\frac{x^{2}}{\nu}\right)^{-(1+\nu) / 2} g(y) d x d y \\
& =\frac{4}{\sqrt{\nu} \mathrm{B}(\nu / 2,1 / 2)} \int_{0}^{\infty} \int_{0}^{\infty} \cos (t x y)\left(1+\frac{x^{2}}{\nu}\right)^{-(1+\nu) / 2} g(y) d x d y
\end{aligned}
$$

The inner integral in (9) can be calculated by direct application of equation (2.5.6.4) in [4, v. 1]. This yields

$$
\phi(t)=\frac{2^{2-\nu / 2} \nu^{\nu / 4} t^{\nu / 2}}{\Gamma(\nu / 2)} \int_{0}^{\infty} y^{\nu / 2} K_{-\nu / 2}(t \sqrt{\nu} y) g(y) d y
$$

For the form of $g(\cdot)$ given by (8), direct application of equation $(2.16 .3 .13)$ in $[4$, v. 2] shows that (10) can be calculated as

$$
\phi(t)=\frac{2^{2-\nu / 2} \nu^{\nu / 4} b^{b / 2} t^{\nu / 2}}{\Gamma(\nu / 2) \mathrm{B}(b / 2,1 / 2)} \int_{0}^{\infty} y^{\nu / 2} K_{-\nu / 2}(t \sqrt{\nu} y)\left(y^{2}+b\right)^{-(1+b) / 2} d y
$$




$$
\begin{aligned}
& =\frac{2^{-\nu / 2} \nu^{\nu / 4} b^{b / 2} t^{\nu / 2}}{\Gamma(\nu / 2) \mathrm{B}(b / 2,1 / 2)} \\
& \times\left[2^{-\nu / 2}(t \sqrt{\nu})^{\nu / 2}(\sqrt{b})^{\nu-b} \Gamma\left(-\frac{\nu}{2}\right) \mathrm{B}\left(\frac{b-\nu}{2}, \frac{\nu+1}{2}\right)\right. \\
& \times_{1} F_{2}\left(\frac{\nu+1}{2} ; 1+\frac{\nu}{2}, 1+\frac{\nu-b}{2} ;-\frac{t^{2} \nu b}{4}\right) \\
& +2^{\nu / 2}(t \sqrt{\nu})^{-\nu / 2}(\sqrt{b})^{-b} \Gamma\left(\frac{\nu}{2}\right) \mathrm{B}\left(\frac{b}{2}, \frac{1}{2}\right){ }_{1} F_{2}\left(\frac{1}{2} ; 1-\frac{\nu}{2}, 1-\frac{b}{2} ;-\frac{t^{2} \nu b}{4}\right) \\
& +2^{\nu / 2-b-2}(t \sqrt{\nu})^{b-\nu / 2} \Gamma\left(-\frac{b}{2}\right) \Gamma\left(\frac{a-b}{2}\right) \\
& \left.\times{ }_{1} F_{2}\left(\frac{1+b}{2} ; 1+\frac{b}{2}, 1+\frac{b-\nu}{2} ;-\frac{t^{2} \nu b}{4}\right)\right] .
\end{aligned}
$$

4. Laplace $g$. If $g$ is the Laplace pdf given by

$$
g(y)=\frac{\lambda}{2} \exp (-\lambda|y-\theta|)
$$

then (2) can be reduced as

$$
\begin{aligned}
\phi(t)=\frac{\lambda \nu^{\nu / 2}}{2 \mathrm{~B}(\nu / 2,1 / 2)} \int_{-\infty}^{\infty} \int_{-\infty}^{\infty} \exp \{i t x y-\lambda|x-\theta|\}\left(y^{2}+\nu\right)^{-(1+\nu) / 2} d x d y \\
=\frac{\lambda \nu^{\nu / 2}}{2 \mathrm{~B}(\nu / 2,1 / 2)} \int_{-\infty}^{\infty}\left(y^{2}+\nu\right)^{-(1+\nu) / 2}\left[\int_{-\infty}^{\theta} \exp \{-\lambda(\theta-x)+i t x y\} d x\right. \\
\left.+\int_{\theta}^{\infty} \exp \{-\lambda(x-\theta)+i t x y\} d x\right] d y \\
=\frac{\lambda \nu^{\nu / 2}}{2 \mathrm{~B}(\nu / 2,1 / 2)} \int_{-\infty}^{\infty}\left(y^{2}+\nu\right)^{-(1+\nu) / 2}\left[\frac{\exp (i t \theta y)}{\lambda+i t y}+\frac{\exp (i t \theta y)}{\lambda-i t y}\right] d y .
\end{aligned}
$$

The integral in (12) cannot be calculated in its general form. However, in the particular case $\nu=1$, one can reduce (12) as

$$
\begin{aligned}
\phi(t) & =\frac{\lambda}{2 \pi} \int_{-\infty}^{\infty}\left(y^{2}+1\right)^{-1}\left[\frac{\exp (i t \theta y)}{\lambda+i t y}+\frac{\exp (i t \theta y)}{\lambda-i t y}\right] d y \\
& =\frac{\lambda}{2 \pi t}\left[\int_{-\infty}^{\infty} \frac{\exp (i t \theta y)}{\left(y^{2}+1\right)(\lambda / t+i y)} d y+\int_{-\infty}^{\infty} \frac{\exp (i t \theta y)}{\left(y^{2}+1\right)(\lambda / t-i y)} d y\right] \\
& =\frac{\lambda}{2}\left[\frac{\exp (t \theta)}{\lambda+t}+\frac{\exp (-t \theta)}{\lambda-t}\right],
\end{aligned}
$$

where the last step follows by direct application of equation $(2.3 .8 .4)$ in $[4$, v. 1].

5. Logistic $g$. Suppose $g$ is the logistic pdf given by

$$
g(y)=\frac{\lambda \exp \{-\lambda(y-\theta)\}}{[1+\exp \{-\lambda(y-\theta)\}]^{2}} \text {. }
$$

Note that this can be reexpressed as the mixture of Laplace pdfs:

$$
g(y)=\sum_{k=0}^{\infty} \frac{2}{k+1}\left(\begin{array}{c}
-2 \\
k
\end{array}\right) \frac{\lambda(k+1)}{2} \exp \{-\lambda(k+1)|y-\theta|\} .
$$

Thus, using the result for the Laplace distribution given by (13), one obtains

$$
\phi(t)=\lambda \sum_{k=0}^{\infty}\left(\begin{array}{c}
-2 \\
k
\end{array}\right)\left[\frac{\exp (t \theta)}{(k+1) \lambda+t}+\frac{\exp (-t \theta)}{(k+1) \lambda-t}\right]
$$

for the particular case $\nu=1$. 
6. Bessel function $g$. Suppose $g$ is the Bessel function pdf given by

$$
g(y)=\frac{|y|^{m}}{\sqrt{\pi} 2^{m} b^{m+1} \Gamma(m+1 / 2)} K_{m}\left(\left|\frac{y}{b}\right|\right) .
$$

For this form, (10) can be expressed as

$$
\phi(t)=\frac{2^{2-\nu / 2} \nu^{\nu / 4} t^{\nu / 2}}{\sqrt{\pi} 2^{m} b^{m+1} \Gamma(\nu / 2) \Gamma(m+1 / 2)} \int_{0}^{\infty} y^{\nu / 2+m} K_{-\nu / 2}(t \sqrt{\nu} y) K_{m}\left(\frac{y}{b}\right) d y .
$$

An application of equation (2.16.33.1) in [4, v. 2] shows that the above can be reduced to

$$
\begin{aligned}
\phi(t)= & \frac{\Gamma((\nu+1) / 2) \Gamma(m+(\nu+1) / 2)}{b^{m+1} \Gamma(\nu / 2) \Gamma(\nu / 2+m+1)} \\
& \times{ }_{2} F_{1}\left(m+\frac{1}{2}, m+\frac{\nu+1}{2} ; \frac{\nu}{2}+m+1 ; 1-t^{2} \nu b^{2}\right) .
\end{aligned}
$$

Using special properties of the Gauss hypergeometric function, one can reduce (15) to elementary forms when $m$ and $\nu$ take integer or half-integer values. For example, if $\nu=1$ and $m=2,3,4,5,6$, then (15) can be reduced to the simple forms

$$
\begin{aligned}
& \phi(t)=\frac{16}{15 \pi}\left\{\frac{25 x-15}{8 x^{2}(x-1)^{2}}+\frac{15 \operatorname{arcth}(\sqrt{x})}{8 x^{5 / 2}}\right\}, \\
& \phi(t)=\frac{32}{35 \pi}\left\{\frac{-231 x^{2}+280 x-105}{48 x^{3}(x-1)^{3}}-\frac{35 \operatorname{arcth}(\sqrt{x})}{16 x^{7 / 2}}\right\}, \\
& \phi(t)=\frac{256}{315 \pi}\left\{\frac{837 x^{3}-1533 x^{2}+1155 x-315}{128 x^{4}(x-1)^{4}}+\frac{315 \operatorname{arcth}(\sqrt{x})}{128 x^{9 / 2}}\right\}, \\
& \phi(t)=\frac{512}{693 \pi}\left\{\frac{-10615 x^{4}+26070 x^{3}-29568 x^{2}+16170 x-3465}{1280(x-1)^{5} x^{5}}-\frac{693 \operatorname{arcth}(\sqrt{x})}{256 x^{11 / 2}}\right\}, \\
& \left.\quad+\frac{3003 \operatorname{arcth}(\sqrt{x})}{1024 x^{13 / 2}}\right\},
\end{aligned}
$$

where $x=1-t^{2} b^{2}$. Also, if $\nu=1$ and $m=\frac{3}{2}, \frac{5}{2}, \frac{7}{2}, \frac{9}{2}, \frac{11}{2}$, then (15) reduces to

$$
\begin{aligned}
& \phi(t)=\frac{1}{x^{2}}+\frac{(12 x-8) \sqrt{1-x}}{8 x^{2}(-1+x)^{2}}, \\
& \phi(t)=-\frac{1}{x^{3}}+\frac{\left(-30 x^{2}+40 x-16\right) \sqrt{1-x}}{16 x^{3}(-1+x)^{3}}, \\
& \phi(t)=\frac{1}{x^{4}}+\frac{\left(280 x^{3}-560 x^{2}+448 x-128\right) \sqrt{1-x}}{128 x^{4}(x-1)^{4}}, \\
& \phi(t)=-\frac{1}{x^{5}}+\frac{\left(-630 x^{4}+1680 x^{3}-2016 x^{2}+1152 x-256\right) \sqrt{1-x}}{256 x^{5}(x-1)^{5}}, \\
& \phi(t)=\frac{1}{x^{6}}+\frac{\left(2772 x^{5}-9240 x^{4}+14784 x^{3}-12672 x^{2}+5632 x-1024\right) \sqrt{1-x}}{1024 x^{6}(x-1)^{6}},
\end{aligned}
$$

where $x=1-t^{2} b^{2}$. 


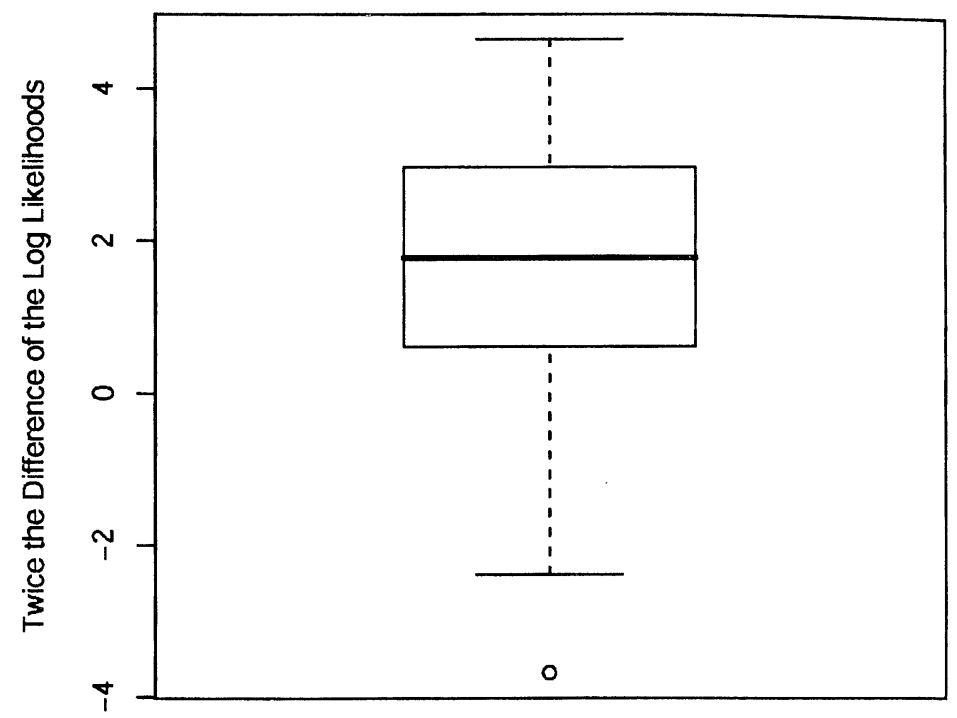

Fig. 1. Box plot of the values of $2\left(\ln L_{2}-\ln L_{1}\right)$ for the 100 simulated samples (each of size 10) from the standard normal distribution

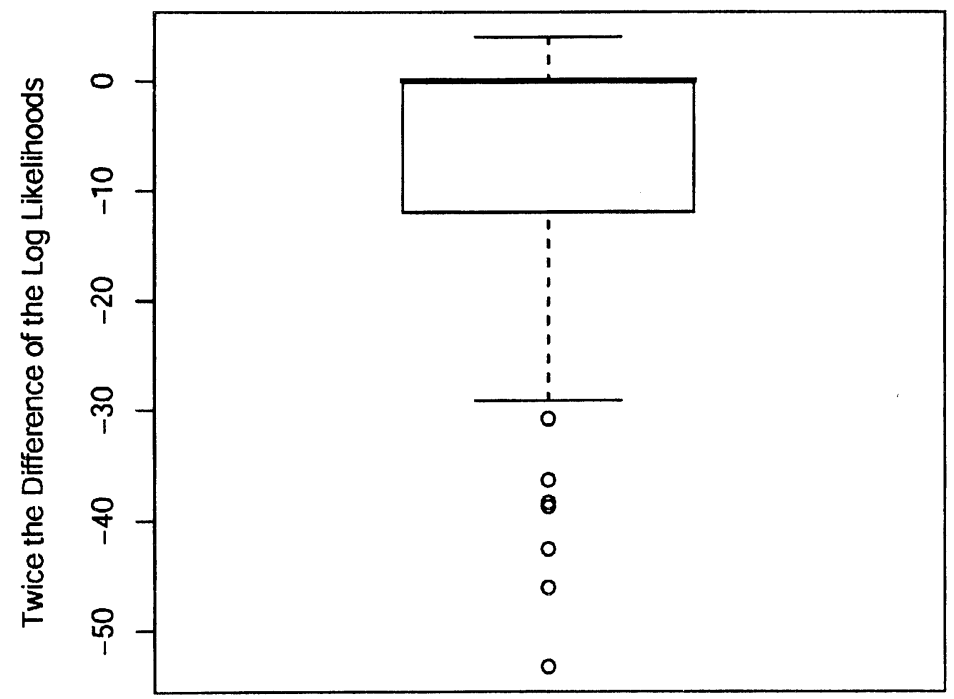

Fig. 2. Box plot of the values of $2\left(\ln L_{2}-\ln L_{1}\right)$ for the 100 simulated samples (each of size 10) from the Student's $t$ distribution with degrees of freedom $\nu=1$ 


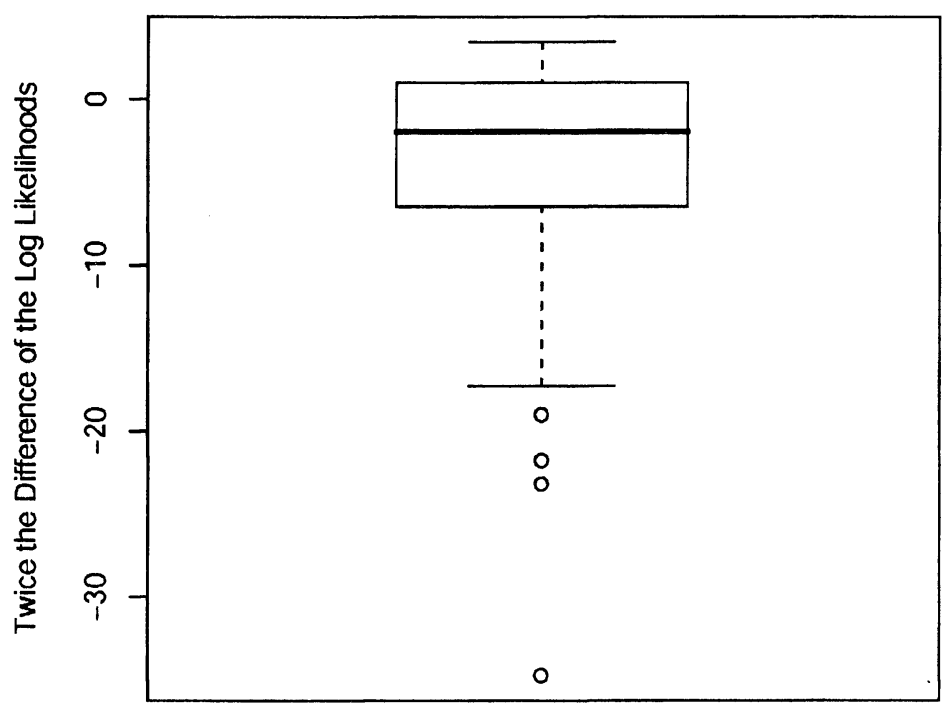

Fig. 3. Box plot of the values of $2\left(\ln L_{2}-\ln L_{1}\right)$ for the 100 simulated samples (each of size 10) from the standard logistic distribution

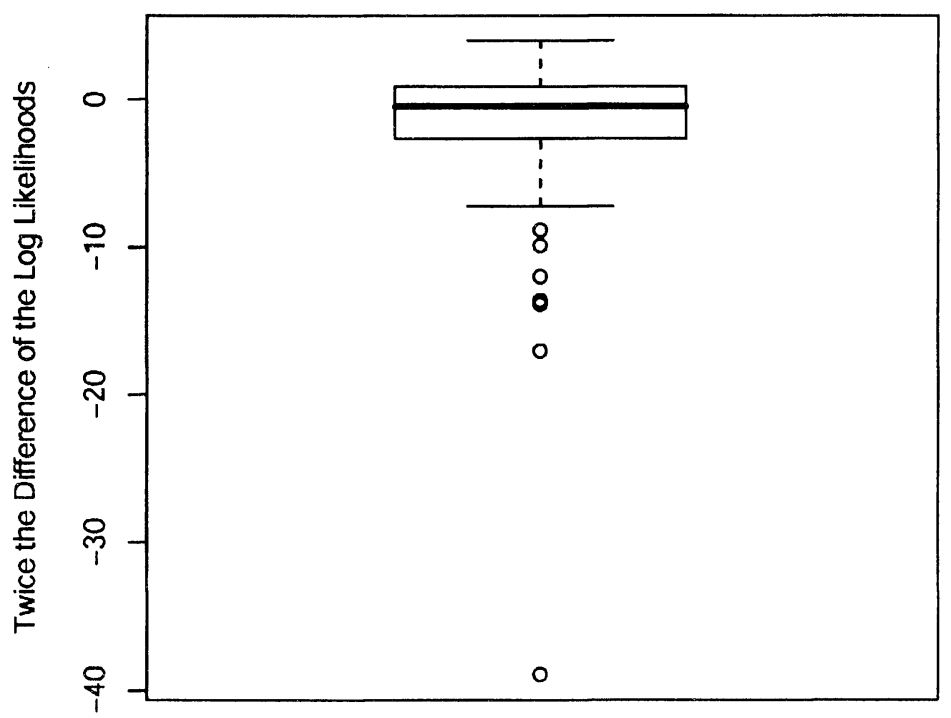

Fig. 4. Box plot of the values of $2\left(\ln L_{2}-\ln L_{1}\right)$ for the 100 simulated samples (each of size 10) from the standard Laplace distribution

7. Application. Recently, various Bayesian approaches have been proposed for DCT coefficient estimation. These approaches impose a prior distribution on the variability/precision of the observed DCT coefficients. The prior model is designed to capture 
the sparseness of DCT coefficient expansions. Then, the image is estimated by applying a suitable Bayesian rule to the resulting posterior distribution of the DCT coefficients. Different choices of loss function lead to different Bayesian rules and hence to different rules for DCT coefficients.

For the reasons mentioned in Section 1, we assume the Student's $t$ prior for the variability/precision of the observed DCT coefficients. It is also assumed in the prior model that the DCT coefficients of the true image are mutually independent random variables and independent of the noise process. Different posterior models for the DCT coefficients can be obtained by assuming different distributions for the noise process. For instance, if we assume that the noise process is white noise, then the posterior will be given by (6).

The most popular model for DCT coefficients has been the normal distribution. However, it has been argued that the normal distribution may not be the best model for every type of image. For example, the work [5] suggested using the Laplace distribution for certain images. Other nonnormal distributions have also been used, see, e.g., [3].

Here, we show that the distribution given by (6) is flexible enough to model the DCT coefficients whether they follow the normal distribution or not. To show this, we simulated 100 samples each of size 10 from each of the following distributions:

1) the standard normal distribution (Model 1);

2) the Student's $t$ distribution with degrees of freedom $\nu=1$ (Model 2);

$3)$ the standard logistic distribution given by the pdf $f(x)=\exp (-x) /\{1+\exp (-x)\}^{2}$ for $-\infty<x<\infty$ (Model 3);

4) the standard Laplace distribution given by the pdf $f(x)=(1 / 2) \exp (-|x|)$ for $-\infty<x<\infty$ (Model 4).

For each of the $100 \times 4=400$ samples, we fitted the distribution given by (6) as well as the standard normal distribution. We computed $2\left(\ln L_{2}-\ln L_{1}\right)$ for each fit, where $L_{1}$ and $L_{2}$ denote the maximized likelihoods for the two distributions. Fig. $k$ shows the box plot of the values of $2\left(\ln L_{2}-\ln L_{1}\right)$ for the 100 samples from Model $k, k=1,2,3,4$.

It is obvious from the figures that the model given by (6) performs as well as the normal distribution when the DCT coefficients are in fact normally distributed, see Fig. 1. More importantly, when the DCT coefficients are not normally distributed (6) performs much better when compared to the normal model, see Figs. 2-4. Thus, (6) is a versatile model whether the DCT coefficients are normally distributed or not.

\section{REFERENCES}

1. Градштейн И.С., Рыжик И.М. Таблицы интегралов, сумм, рядов и произведений. М.: Наука, 1971, 1108 с.

2. Kotz S., Nadarajah $S$. Multivariate $t$ distributions and their applications. Cambridge: Cambridge Univ. Press, 2004, 272 p.

3. Lam E. Y. Analysis of the DCT coefficient distributions for document coding. - IEEE Signal Processing Lett., 2004, v. 11, № 2, p. 97-100.

4. Прудников А.П., Брычков Ю.А., Маричев О.И. Интегралы и ряды, т. 1-3. М.: Физматлит, 2003, $631 \mathrm{c} ; 664 \mathrm{c} ; 687 \mathrm{c.}$

5. Reininger R.C., Gibson J.D. Distribution of the two-dimensional DCT coefficients for images. - IEEE Trans. Commun., 1983, v. 31, p. 835-839.

Поступила в редакцию

6.I. 2005

Исправленный вариант

20.XII. 2007 